\title{
Itinéraires
}

Itinéraires Littérature, textes, cultures

2010-3 | 2010

Médiévalisme

\section{Médiévalismes d'une sémiose : le Moyen Âge en chanson}

Céline Cecchetto

\section{(2) OpenEdition}

Journals

\section{Édition électronique}

URL : http://journals.openedition.org/itineraires/1905

DOI : $10.4000 /$ itineraires. 1905

ISSN : 2427-920X

Éditeur

Pléiade

\section{Édition imprimée}

Date de publication : 1 novembre 2010

Pagination : 177-188

ISBN : 978-2-296-13150-7

ISSN : $2100-1340$

Référence électronique

Céline Cecchetto, "Médiévalismes d'une sémiose : le Moyen Âge en chanson », Itinéraires [En ligne], 2010-3 | 2010, mis en ligne le 01 novembre 2010, consulté le 06 mai 2019. URL : http://

journals.openedition.org/itineraires/1905; DOI : 10.4000/itineraires.1905

\section{(ब) $(\Theta \Theta$}

Itinéraires est mis à disposition selon les termes de la licence Creative Commons Attribution - Pas d'Utilisation Commerciale - Pas de Modification 4.0 International. 


\title{
Médiévalismes d'une sémiose : le Moyen Âge en chanson
}

\begin{abstract}
Reminiscences of the Middle Ages in the contemporary french song do not concern only the adaptation of medieval texts. Such eminently memorial genre, uses a combination of various semiotic networks (text, music, orchestration, voice, imagery, presentation...) that is necessary to study together to understand its meaning. How can the theory of intertextuality work for musical, pictural and performance analysis? The development of these tools ultimately allows to overcome the issue of reference, to think about the Middle Ages as a network of signs giving shape to a collective memory.
\end{abstract}

Keywords : song, musique, memory, intertextuality, semiotics

Mots clés : chanson, musique, mémoire, intertextualité, sémiotique

À la recherche d'une épaisseur théorique pour le concept de médiévalisme, on ne saurait oublier le domaine de la chanson, trop souvent traité avec légèreté. Michel Zink se demandait, comme pour introduire ce questionnement :

Pourquoi le goût des vieilles chansons? Pourquoi chercher dans le Moyen Âge les racines des chansons françaises? Pourquoi, à l'époque moderne, certaines chansons nous renvoient-elles au Moyen Âge ${ }^{1}$ ?

Après un parcours au cœur des échos du Moyen Âge dans la chanson contemporaine entrepris voilà plusieurs années ${ }^{2}$, nous voudrions aujourd'hui proposer une modeste synthèse théorique, et quelques outils méthodologiques qui permettent d'éclairer la notion de médiévalisme à la lumière de la combinatoire. La chanson, en effet, attire aujourd'hui

1. Michel Zink, Le Moyen Âge et ses chansons, Paris, De Fallois, 1996, p. 55.

2. Cf. Céline Cecchetto, Échos du Moyen Âge et de la Renaissance dans la chanson française contemporaine, thèse de doctorat, Université de Bordeaux III, 2008. 
l'attention des critiques non comme sous-genre littéraire, mais bien comme catégorie architextuelle fonctionnant comme sémiose, c'est-à-dire comme « combinaison de plusieurs systèmes signifiants en un seul espace, concourant à produire un sens unitaire ${ }^{3} \gg$ : texte, musique, voix, interprétation, orchestration, iconographie, scénographie. En un mot, c'est à l'aune de la cantologie qu'il faut étudier aujourd'hui la chanson. Elle intéresse particulièrement celui qui questionne la notion de médiévalisme, puisque selon les critères cantologiques, ce n'est donc pas dans les mots et leur agencement que réside une possible poésie propre aux œuvres écrites pour la chanson, mais plutôt « dans la dynamique de questionnement temporel qu'instaure la double articulation de ces mots avec d'une part la musique qui les porte, et d'autre part l'interprète qui choisit de les incarner ${ }^{4} »$.

Il faut donc partir à la rencontre des signes médiévaux et considérer le Moyen Âge comme une catégorie sémiologique. Plus largement, en l'étudiant comme champ sémiotique (ce que permet la chanson) on peut révéler sa spécificité culturelle, épistémologique et enfin, critique : la validation d'une transposition critique du texte/tissu médiéval comme spécificité d'un regard sur le monde n'intervient qu'après ce point de départ. Pour questionner l'existence d'un médiévalisme chansonnier, peut-être ne suffit-il pas cependant d'entreprendre un repérage des traces plus ou moins claires du Moyen Âge. La pertinence du médiévalisme appliqué à la chanson réside également dans la prégnance de certains concepts médiévaux qui permettent de parfaire un discours sur le genre chanson: comment montrer, à partir d'une étude du médiévalisme en chanson que la spécificité de la chanson est bien de donner forme à une mémoire collective? Pour répondre à cette question, non seulement la notion de médiévalisme devra avoir été étayée par les diverses couches sémiotiques à l'œuvre dans la chanson, mais elle deviendra à son tour outil de conceptualisation pour la critique émergente de ce genre de discours. Il s'agira donc de repérer le fait médiéval tel qu'il se présente en chanson. Pour éviter l'écueil d'une induction faussée, une solution est de décliner la théorie intertextuelle telle que la propose Genette à l'ensemble des champs sémiologiques, en s'attachant à rendre cohérente et unifiée la signifiance de l'analyse, qui ne doit pas se limiter au fractionnement des champs sémiologiques.

Le degré de présence du Moyen Âge dans la chanson contemporaine ne définit pas une catégorie d'œuvres à grande visibilité, en raison de la grande disparité stylistique et temporelle des pièces concernées. Pourtant, le repérage d'un ensemble de signes médiévaux permet de donner corps

3. Paul Zumthor, Introduction à la poésie orale, Paris, Seuil, 1983, p. 184.

4. Formule empruntée à une conférence de Stéphane Hirschi, qui a mis au point la théorie de la cantologie (« De la chanson comme poésie des mots incarnée », conférence prononcée lors des troisièmes Semaines de Musique Française, AFPC, 1999). 
à une forme originale de médiévalisme. Mais le chercheur en cantologie ne dispose pas d'outil stable et efficace à cette fin, pour deux raisons principales : d'une part, la critique contemporaine considère difficilement la chanson comme un objet d'étude autre que sociologique; d'autre part, la diversité des réseaux signifiants mis à contribution dans la production du sens empêche la simple utilisation d'un concept préexistant. On a donc fonctionné par extensions successives d'un modèle donné, partant du texte pour embrasser l'ensemble du tissu chansonnier.

\section{Intertextes et hypertextes médiévaux}

La chanson, bien qu'objet performatif et performanciel, demeure culturellement marquée par son empreinte textuelle, qui constitua longtemps le maître étalon de sa valeur artistique. Les catégories de Genette restent ainsi parfaitement (et classiquement) opératoires pour repérer les traces médiévales : les phénomènes de citations et d'hypertextualité s'affirment ainsi chez un très petit nombre d'auteurs de chansons, au premier rang desquels Georges Brassens. Leur rapport au Moyen Âge reste marqué par une certaine érudition. Dans le répertoire de Brassens, Le Moyenâgeux s'articule au Testament comme le pastiche à la parodie. Une incursion dans le répertoire québecois, chez Richard Desjardins, suffit à montrer le médiévalisme villonien à l'œuvre dans Lomer, qui pastiche et parodie à la fois le Testament de Villon :

Adieu mon frère, adieu ma sœur demain à l'aube les pieds nus, j'irai dans les vastes noirceurs d'où personne n'est revenu.

Adieu la Terre, tant si bonne, qui tant d'eau froide m'a fait boire.

Adieu Humains, qu'on me pardonne si je ne laisse que mon histoire.

En l'an quarantième de mon âge, hors d'enfance et franc de dettes, pourvu de sens, du moins le crois-je, nul méfait que je regrette.

Qui meurt a ses lois de tout dire.

Écoutez bien, honnêtes gens, car on m'a jugé à mourir.

Je me tais et je commence ${ }^{5}$.

Des signes plus simplement linguistiques concourent à produire un médiévalisme chansonnier par divers emprunts à l'ancien français, 
le Moyen Âge étant perçu comme un ensemble de références précises, signifiées par des « marqueurs lexicaux ${ }^{6}$ », ainsi que les nomme Danièle James Raoul, comme l'est le mot « Oyez », chez Pierre Delanoé :

Oyez, oyez, oyez,

C'est la chanson yéyé ${ }^{7}$

ou Zazie :

Oyez oyez braves gens $[\ldots]$

Oyez oyez les misters ${ }^{8}$.

Langue et littérature médiévales concourent ainsi à jeter les bases d'un univers médiéval qui peut fonctionner comme un système cohérent (chez R. Desjardins) ou se distiller en touches frappantes qui ramènent l'auditeur à un mode ancien du rapport à l'oralité.

Cette approche textuelle des effets de sens dans la chanson constitue l'essentiel des études chansonnières $\mathrm{du} \mathrm{xx}^{\mathrm{e}}$ siècle. On a souvent jugé bon de la seconder par une analyse musicologique de l'«air» de la chanson (c'est-à-dire sa mélodie). Malgré l'apparent intérêt offert par ce complément d'analyse, le secours de la musicologie se contentait trop souvent de démontrer comment la musique soutenait le texte, et donc l'effet médiéval, plutôt que de montrer comment elle fonctionnait comme réseau sémiologique et sémiotique à part entière. L'approche strictement intertextuelle ou hypertextuelle n'est donc opérante que dans la mesure où elle correspond à un certain mode d'appréhension de composition de la chanson : celle qui en fait un dérivé de la poésie, uniquement sauvée de la paralittérature par un de ses sous-genres, la « chanson poétique ${ }^{9}$ ». On sait aujourd'hui que pour considérer la spécificité du mode de communication chansonnier, la chanson, fût-elle une "chanson de variété », ne doit plus être pensée en ces termes. Lorsque l'on cherche à comprendre le fonctionnement du genre chanson, l'insuffisance de la seule approche textuelle se révèle à la lumière de la cantologie.

6. Danièle James-Raoul, «Moult à travers les âges ( $\mathrm{XVI}^{\mathrm{e}}-\mathrm{XXI}^{\mathrm{e}}$ siècles) : de la désuétude à la résurrection ", dans Claire Badiou-Monferran, Frédéric Calas, Julien Piat et al., La langue, le style, le sens. Études offertes à Anne-Marie Garagnon, Paris, L'improviste, 2006, p. 133.

7. Je ne sais pas comment, texte de Pierre Delanoé, dans Pierre Delanoé, Des paroles qui chantent, Paris, Christian Pirot, 1997, p. 24.

8. Zazie, Avis au sexe fort (Zazie), 1992.

9. Voir Brigitte Buffard-Moret, «Le statut de la chanson au sein de la poésie française (XVI ${ }^{\mathrm{e}}-\mathrm{XIX}^{\mathrm{e}}$ siècles) », dans Claire Badiou-Monferran, Frédéric Calas, Julien Piat et al., op. cit., p. 377-390. 
La spécificité du médiévalisme chansonnier ne peut donc se limiter à la recherche des traces du Moyen Âge dans les effets intertextuels et hypertextuels et une telle attitude conduit à deux écueils majeurs : d'une part on méconnaît le fonctionnement du genre chanson, qui, depuis le Moyen Âge, trouve sa signifiance dans la conjonction de réseaux sémiotiques; d'autre part, la prévalence de l'ensemble textuel dans le système de valeur de la culture occidentale peut également grever la création chansonnière lorsqu'elle veut produire un effet médiéval: la teneur du signe médiéval y perd de sa puissance. En effet, si la spécificité du signe linguistique médiéval est bien d'être d'un accès difficile pour des non spécialistes, alors les médiévalismes langagiers circonscrivent ce signe à un simple « exotisme » singulier qui n'a de valeur que par son étrangeté. Lorsque Bourvil chante, en 1959, Les Rois fainéants, le médiévalisme s'apparente clairement à un amas de médiévotypes lexicaux :

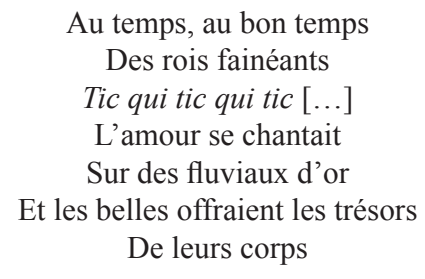

Aux rois fainéants.

Les baladins jouaient du bilboquet

Et les ménestrels grattaient leurs vielles

Et tous les bouffons dansaient en rond :

C'était toujours la fête!

Tous les manants, badauds criaient

«Bravo!»

«Foutez-moi la paix!»

Au bord des ruisseaux,

Je te vois, quenouillant le lin [...]

\begin{abstract}
Alors mon amour
$\mathrm{Ne}$ crie pas toujours

Laisse-moi dormir

Et sourire ${ }^{10}$.
\end{abstract}

L'ambiance médiévale de la chanson est un effet de style destiné à parfaire la construction d'un univers étroitement associé à la spécificité de l'interprète. Ce dernier endosse la fonction de fantaisiste : diction abêtie, musique marquée par des figuralismes, mise en scène finale du chanteur en « fainéant » concourent à transformer la chanson en saynète humoristique. Le choix d'un lexique moyenâgeux est le prétexte à la déclinaison de la «marque de fabrique » Bourvil, synthétisée dans le «ti qui tic qui tic» 
récurrent. Le médiévalisme se réduit donc à un procédé de mise à distance exotique visant à mettre en avant la personnalité du chanteur ${ }^{11}$.

Le fonctionnement du signe transtextuel médiéval en chanson entrant en résonnance avec un genre tout entier fondé sur la mémoire, on entrevoit donc la spécificité du médiévalisme en chanson: la chanson n'est pas le champ générique d'une recréation mais d'un souvenir, parce qu'elle fonctionne elle-même sur le schème de la "fixation d'un air ${ }^{12}$ ». La théorie intertextuelle permet ainsi de penser la signification d'un possible médiévalisme, à condition que ce dernier soit à la fois sémiotique et cantologique : il faut donc trouver les moyens de l'étendre aux autres champs de signification du genre.

\section{Extensions des catégories intertextuelles}

À la lumière de la théorie cantologique, on peut alors aborder la notion de médiévalisme en cherchant dans la forge genettienne l'extension polysémiotique de ses théories.

La première touche évidemment le champ musical. Nous lui avons donné le nom d'intermusicalité. Le fondement épistémologique de cet outil d'analyse mêle la théorie intertextuelle et les théories d'analyse anglo-saxonnes des popular music, notamment celles de Philip Tagg et Gary Burns ${ }^{13}$. L'intermusicalité peut être définie comme la présence effective dans une pièce musicale d'un signe musical, techniquement défini comme appartenant à un des différents « aspects » musicaux de la musique populaire définis par Philipp Tagg, et renvoyant structurellement à un « horizon » différent de celui dans lequel est placé l'énonciateur actuel. Gary Burns étaye quant à lui l'approche intertextuelle pour l'étude des réminiscences médiévales dans la chanson d'aujourd'hui. Selon lui les musiques populaires contemporaines sont en effet constituées d'un agencement de hooks (que le français traduit mal par « accroches »), des éléments d'une chanson qui restent en mémoire parce qu'ils sont l'objet d'une répétition. Ils donnent à un morceau sa spécificité, sa personnalité. La typologie qu'il en établit se constitue en fonction de critères relatifs au rythme, à la mélodie, à l'harmonie, aux paroles, au dispositif instrumental, au tempo, à l'intensité, à l'improvisation, aux effets sonores, à l'editing, au mixage, à l'équilibre relatif entre sources d'émission et à la distorsion du signal sonore. Grâce au repérage de ces accroches, Giancarlo Siciliano explique comment on peut penser les traces musicales en termes d'intertextualité, en interprétant les hooks comme des « intertextèmes » :

11. Cela concourt à insérer l'interprète dans le système économique de la chanson : aisément identifiable, il agit comme facteur de vente potentiel.

12. Stéphane Hirschi définit la chanson comme un « air fixé par des paroles » (op. cit.).

13. Philip Tagg, «Analysing popular music », Popular Music, $\mathrm{n}^{\circ} 2$, 1982, p. 37-65; Gary Burns, « A typology of hooks in popular music », Popular Music, n 6/1, 1987, p. 1-20. 
Les principaux intertextèmes se constituent en fonction de critères relatifs à la typologie de Gary Burns. [...] En souscrivant à la logique selon laquelle tout texte «se construit comme mosaïque de citations, [...] absorption et transformation d'un autre texte » (Kristeva, 1969, p. 85), la notion d'intertextualité trouve une matérialisation sonore dans la complexe topologie du fait musical contemporain. Si les musiques européennes de tradition classique ne se laissent pas réduire à un Urtext (texte original), leurs contreparties non-classiques, elles, se transforment en permanence en hypertextes, au sens métapoétique de Gérard Genette ${ }^{14}$.

Comment rendre opérante cette nouvelle catégorie dans la recherche des médiévalismes? Il fallait prendre en compte la spécificité du signe musical qui n'est pas tout à fait celle du signe saussurien. Les termes qu'emploie le musicologue Jean-Jacques Nattiez pour la définir semblent tout naturellement inviter à penser le Moyen Âge moins comme un territoire (ce qu'il peut être dans d'autres genres, d'autres pratiques) que comme un horizon :

un objet prend une signification pour un individu qui l'appréhende lorsqu'il met cet objet en relation avec des secteurs de son vécu, c'est-à-dire l'ensemble des autres objets qui appartiennent à son expérience du monde. Dans une formulation plus lapidaire : il y a signification quand un objet est mis en relation avec un horizon ${ }^{15}$.

Pour cerner cet horizon, on peut scinder en autant d'aspects les territoires musicaux touchés par l'empreinte du Moyen Âge :

- L'instrumentarium: le choix d'instruments anciens dans la performance chantée fonctionne comme signe immédiat de la médiévalité, en particulier chez des interprètes visant à produire des œuvres marquées du sceau de la tradition et de l'intemporalité. Ainsi, le groupe Malicorne utilise-t-il dulcimer, épinette des Vosges, vielle à roue et orgue positif ${ }^{16}$.

- L'utilisation de la modalité : les modes grégoriens continuent à être utilisés par certains compositeurs en vue de produire un effet antiquisant. Pour le groupe Pigalle, par exemple, on note dans la chanson Premières Fois l'utilisation des modes de Mi et La, avec transitions, repos sur la plagale et la finale :

14. Giancarlo Siciliano, « Musique et postmodernité :pratiques/théorisations/interférences », Trans, $\mathrm{n}^{\circ}$ 3, 1997 (http://www.sibetrans.com/trans/trans3/siciliano.htm).

15. Jean-Jacques Nattiez, «La signification comme paramètre musical », dans Musiques, vol. II, Arles, Actes Sud, Paris, Cité de la musique, 2004, p. 256. Voir sur ce point les travaux de Serge Lacasse sur la notion de transphonographie qui invitent aujourd'hui à montrer pourquoi la théorie intertextuelle peut avoir une existence polysémiotique au sein d'un genre particulier (Serge Lacasse, "Composition, performance, phonographie : un malentendu ontologique en analyse musicale? », dans Serge Lacasse et Patrick Roy (dir.), "Une étoile qui danse »: Mélanges à la mémoire de Roger Chamberland, Québec, Presses de l'Université Laval, 2005).

16. Il s'agit de l'instrumentarium de l'album Hexagone, paru en 1975 (Polydor). 


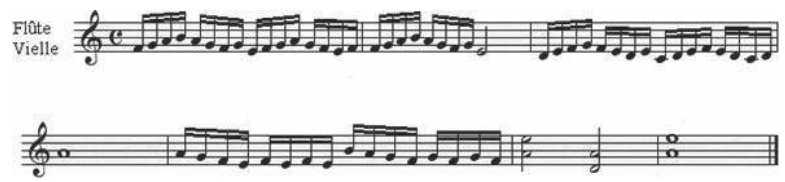

- L'utilisation des modes rythmiques: les aspects rythmiques de l'intermusicalité médiévale concernent d'abord les recours aux modes rythmiques anciens. Jean Beck distingue en effet deux sortes de modes à deux éléments, tous deux ternaires et formés de la succession d'une longue de deux temps et d'une brève d'un temps : dans le $1^{\text {er }}$ mode, la longue précède la brève, ce qui répond au pied trochaïque des anciens, dans le $2^{\mathrm{e}}$ mode, la brève précède la longue (pied iambique). Un chanteur tel que Georges Brassens l'utilisa abondamment dans des chansons marquées par le poids du temps (Bonhomme) ou de l'histoire (Le Petit Joueur de fluteau).

- L'utilisation de structures polyphoniques spécifiques : les groupes à dominante vocale, tels Chanson Plus Bifluorée, utilisent volontiers les techniques de la polyphonie médiévale, telle le hoquet ${ }^{17}$, dans des chansons à visée parodique où la mise à distance de l'horizon médiéval invite à apprécier la réflexivité de l'œuvre du groupe.

Mais il faut encore sortir de la dichotomie «paroles et musique» : la cantologie parle bien du genre chanson comme de celui d'une performance chantée, qui doit être étudiée comme espace de signification visuelle. À ce titre, on peut effectivement parler d'interpicturalité, voir d'interperformativité, à prendre en compte les conditions de la performance chantée comme signe d'un possible médiévalisme. L'intérêt critique se double encore d'une mise en lumière des diverses médialités qui concourent à la production d'une image où existe le signe médiéval. Dans les pochettes de disque, les images et looks que se donnent certains chanteurs, on peut aisément repérer comment fonctionne le signe Moyen Âge. Les références aux représentations artistiques médiévales, en particulier, sont autant de signes d'un médiévalisme assumé. On a pu le voir ailleurs pour le «signe » troubadour, distillé chez certains auteurscompositeurs-interprètes pour donner le crédit de l'origine à la qualité de leurs productions ${ }^{18}$. En examinant l'univers pictural d'un groupe tel que Têtes Raides, on repère aisément que le collectif d'art graphique « Les chats pelés » qui prend en charge les illustrations de chacun de leurs albums

17. « Il s'agit d'une technique de contrepoint à plusieurs voix, le plus souvent à deux voix : là où l'une des voix se tait, l'autre est entendue, et inversement, dans une série d'échanges où l'élément de silence vient jouer un rôle important dans l'ensemble rythmique de la polyphonie » (article « Hoquet » dans Françoise Ferrand et Olivier Cullin (dir.), Guide de la musique au Moyen Âge, Paris, Fayard, 1999.

18. Le chanteur du groupe Têtes Raides, Christian Olivier, fait lui-même partie de ce collectif d'artistes 
est largement inspirés des peintres de l'Automne du Moyen Âge. On retrouve dans l'album Le Bout du toit ${ }^{19}$ le foisonnement monstrueux des tableaux d'un Jérôme Bosch, et dans la mise en scène de la performance, en particulier lors de leur tournée aux Bouffes du nord rappellent tout ensemble la profusion et l'éclairage d'un Bruegel ${ }^{20}$.

Les différentes strates de l'imagier médiéval apparaissent alors : les couches de représentations se distillent en autant de signes particuliers qui touchent tous les aspects de la construction de l'image.

\section{Signification d'une trans-sémiotique médiévale}

Pour subsumer l'ensemble de ce processus, on pourra donc enfin cerner la spécificité du médiévalisme en chanson par le terme «transsémiotique médiévale ». L'extension des catégories transtextuelles est absolument nécessaire pour réussir à cerner la présence du Moyen Âge en chanson. Un écueil épistémologique menace pourtant la possibilité même de cette démarche : la segmentation induite par une telle approche semble pousser le chercheur à faire en quelque sorte une " collection » de signes médiévaux, dont il montrerait, certes, le fonctionnement intrinsèque et dont il exploiterait la signification dans l'espace chansonnier. Comment une telle approche peut-elle rendre compte d'une signification cantologique, c'est-à-dire unifiée, d'une chanson donnée? La pensée d'un possible médiévalisme suggère une attitude créatrice : comment rendre compte de la mise en ordre des signes médiévaux pour l'auditeur?

Toute attitude sémiotique d'appréhension du Moyen Âge doit, pour être féconde, donner à lire la façon dont se construit, à partir d'elle, un imaginaire. L'intérêt d'une combinatoire entre la théorie transtextuelle (et ses extensions) et une critique de l'imaginaire est dès lors manifeste et permet au critique d'adopter ce regard englobant qui finit par faire de l'œuvre chantée le vecteur d'un sens, en particulier pour le médiévalisme.

Claude-Gilbert Dubois envisage l'imaginaire comme « le résultat visible d'une énergie formalisée au niveau individuel comme au niveau collectif ${ }^{21} »$. L'énergie : le mouvement et la force, c'est bien ce que l'on tente de saisir de l'espace médiéval dans le genre chanson. L'imaginaire énergique du Moyen Âge peut ainsi se développer dans deux directions majeures, qui reprennent les catégories anthropologiques du même et de l'autre, selon qu'il procède d'un processus d'identification ou de mise à distance. Ainsi, on devra prendre en compte les spécificités

19. Têtes Raides, Le Bout du toit, Tôt ou tard/Wéa, 1996.

20. Voir sur ce point Céline Cecchetto, « Popularité et mémoire dans trois albums de chanson française contemporaine (Têtes Raides, Les Ogres de Barback, Weepers Circus) », Musiques populaires, une exception francophone? Colloque de l'IASPM-branche francophone d'Europe (en ligne : http://iaspmfrancophone.online.fr/colloque2007/).

21. Claude-Gilbert Dubois, L'Imaginaire de la Renaissance, Paris, Seuil, 1985, p.17. 
de la situation d'énonciation de la chanson pour pouvoir cerner le sens du médiévalisme: qui est l'interprète? Comment construit-il médiatiquement sa figure de chanteur? Comment la chanson en question s'inscrit-elle dans son répertoire?

Parmi ceux qui revendiquent une inspiration ou une familiarité certaine avec l'idée de Moyen Âge, certains utilisent donc le médiévalisme pour bâtir leur personnalité d'interprète, sur l'identification à une sémiotique médiévale. Leur image sera donc marquée du sceau du passé et de l'irrémédiablement révolu (pour Georges Brassens, Léo Ferré, Maxime Le forestier, mais également des interprètes plus contemporains : Manau, Têtes Raides, Weepers Circus)... Certains, au contraire, font du Moyen Âge le domaine repoussoir de l'autre, contre lequel se bâtit leur personnalité d'interprète. Bourvil et Chanson Plus Bifluorée, mais également les grandes comédies musicales bâties sur un sujet médiéval (Notre Dame de Paris, Graal) proposent au public une fiction d'interprète non pas tournée vers le passé mais le fixant au contraire dans une série de «médiévotypes »face auxquels ils déploient (ou non) une certaine spécificité créatrice. Les médiévalismes à l'œuvre dans le tissu chansonnier font donc sens dans une économie générale de la communication chansonnière. C'est la pertinence d'un tel concept (le médiévalisme) que l'on doit maintenant évaluer en chanson.

La théorie cognitive de Sperber et Wilson, qui considère la communication comme une modification du contexte de l'énonciation, mesure la pertinence du message à la façon dont celui-ci modifie le contexte d'énonciation par les informations qu'il amène ${ }^{22}$. Une telle approche, pragmatique, invite à penser le médiévalisme en chanson à la lumière de «l'harmonie idéale de la réception» dont participe le genre ${ }^{23}$. Les paramètres de la situation de communication propre à chaque chanson participent ainsi pleinement à la construction d'un médiévalisme réellement signifiant. La pertinence d'une mise en chanson de François Villon n'intervient donc pas dans le même horizon selon qu'elle est le fait de Monique Morelli ${ }^{24}$ ou du Weepers Circus. Pour la première, elle intervient dans un répertoire marqué (du point de vue créateur) par l'écrasante primauté du texte littéraire et (du point de vue du public) par l'inscription dans une famille de chanteurs célébrant la ville et ses marginaux. Le médiévalisme y participe donc d'une identification au "même ", et l'étude sémiologique des mises en chanson montre d'ailleurs que les éléments intermusicaux et interpicturaux renvoient à un code qui n'est pas celui du

22. Dan Sperber et Deirdre Wilson, La Pertinence, communication et cognition, Paris, Minuit, 1989.

23. Voir la définition de Stéphane Hirschi : «l'art du chansonnier réside dans sa capacité d'articuler toutes les composantes de son oeuvre en vue d'une harmonie idéale de la réception » (Jacques Brel, Chant contre silence, Paris, Nizet, 1991).

24. Monique Morelli, Monique Morelli chante Villon, Le chant du monde, 1990. 
Moyen Âge, mais qui vise cependant à mettre en avant les qualités médiévales du texte (tant sur le plan purement linguistique que poétique). Pour le Groupe Weepers Circus, au contraire, la mise en musique de la Ballade de la belle heaumière aux filles de joie prend corps dans une énonciation marquée par le concept de "nouvelle scène ", et qui pour le public et la critique journalistique trouve sa cohérence autour de la valorisation d'un héritage considéré comme passé ${ }^{25}$. À la lumière de ces théories, le médiévalisme chansonnier nous invite à penser le tissu mémoriel du genre chanson dans l'entrelacement même de ses fils et à éviter l'écueil du seul « nostalgique » qui n'est pas suffisamment opératoire ni pour la chanson, ni pour le concept de Moyen Âge.

De ces quelques pistes, la notion de médiévalisme apparait comme épaissie par la teneur sémiologique et sémiotique du genre chanson. Sa cohérence permet la déclinaison de l'outil intertextuel aux divers champs sémiotiques du genre, et donc de penser, en retour, la cohérence de ce dernier. Sa pertinence se développe en effet dans l'étude des signes médiévaux à l'horizon de la réception. Peut-être faut-il alors aller plus avant et considérer ensemble les deux espaces sémiologiques que sont le Moyen Âge et la chanson. Il semble que l'on puisse imaginer un autre terrain d'existence du médiévalisme. Comme ensemble signifiant, le Moyen Âge est une réponse à la modernité par la représentation d'un passé dans une perspective qui ne soit pas historique ${ }^{26}$. Comme architexte, la chanson fonctionne comme un tissu mémoriel ${ }^{27}$. Nous reformulons en ces termes à l'aune médiévale la posture cantologique, pour laquelle une chanson est «un air fixé par des paroles». Pour que la fixation ait lieu, il importe que l'air soit bien mémorisable; deux territoires mémoriels se dessinent donc : celui des œuvres chantées et celui du genre chanson. $\mathrm{Ne}$ pourrait-on pas, dès lors, penser ce dernier à la lumière du Moyen Âge et faire en quelque sorte œuvre de médiévalisme critique? Paul Zumthor affirmait dans l'Essai de poétique médiévale que la poésie médiévale est «le discours d'une mémoire ${ }^{28}$ ». Pourquoi ces mots semblent-ils correspondre si parfaitement au genre chanson tel que la critique la plus récente l'interprète aujourd'hui? L'existence de deux temps parallèles rend donc possible le développement d'une analyse générique nouvelle entièrement pensée depuis le point de vue médiéval :

25. Weepers Circus, Ballade de la belle heaumière aux filles de joie (Villon/Weepers Circus), 1998.

26. Voir dans ce volume les articles de Gil Bartholeyns, Jeff Rider et Thomas Honegger, en particulier.

27. Céline Cecchetto, Échos du Moyen Âge et de la Renaissance dans la chanson française contemporaine, op. cit., chap. II.

28. Paul Zumthor, op. cit., p. 143. 
Erreur de postuler un temps historique absolu : il y a des temps différents bien que parallèles. En ce sens, un des temps de ce qu'on appelle le Moyen Âge peut coïncider avec un des temps de ce qu'on appelle l'Âge Moderne [...]. Dans cet autre temps où tout s'accède à la condition de figure, où tout a une valeur en tant que signe et non en tant que thème descriptif, [des artistes] tentent une œuvre qui peut sembler étrange ou antagonique au temps et à l'histoire qui les environnent, mais qui cependant les inclut, les explique ${ }^{29}$.

Pourquoi? Car la pensée du Moyen Âge est celle de la mise en forme d'une mémoire. Le médiévalisme est aussi la possibilité pour le chercheur de se construire des outils médiévaux pour penser sa propre réalité culturelle, lorsque la pertinence de ses objets d'étude l'y autorise. La rencontre de deux univers mémoriels, l'un, générique, l'autre devenu catégorie de la pensée invite à penser le "passé sans histoire » qu'est le Moyen Âge comme révélant une sémiologie de la mémoire. Un « isthme médiéval » en somme, une fine bande de terre émergée, comme signe d'une mémoire qui serait enfin, dirait Genette, vraiment « révolutionnaire » puisqu'elle « ne se contente[rait] pas de commémorer ${ }^{30} »$.

Céline Cecchetto

Université de Bordeaux 3 - LAPRIL (EA 4198)

29. Julio Cortazár, Marelle [1963], trad. de Laure Guille-Bataillon et Françoise Rosset, Paris, Gallimard, coll. « L'imaginaire », 1966, p. 502.

30. Gérard Genette, Palimpsestes, Paris, Seuil, 1992, p. 453 : «La mémoire est, diton, "révolutionnaire" - à condition qu'on la féconde, et qu'elle ne se contente pas de commémorer». 